\title{
Primary renal carcinoid tumor in horseshoe kidney: a case report
}

\author{
Amira Kamal El-Hawary ${ }^{1}$, Mahmoud Abdo El-Baz ${ }^{1}$, Doaa Sharaf ${ }^{2}$, Ahmed Sobhy El-Hefnawy ${ }^{3}$, Atta \\ Allah Shabaan ${ }^{3}$ \\ 1. Pathology department, Faculty of medicine, Mansoura University, Mansoura, Egypt. 2. Radiology department, Urology \\ and Nephrology Center, Mansoura University, Mansoura, Egypt. 3. Urology department, Urology and Nephrology Center, \\ Mansoura University, Mansoura, Egypt.
}

Correspondence: Amira Kamal El-Hawary. Address: Pathology department, Faculty of medicine, Mansoura University, Mansoura, Egypt. E-mail: Amira960@hotmail.com

Received: February 24, 2014

Accepted: March 19, 2014

Online Published: March 24, 2014

DOI : $10.5430 /$ crcp.v1n2p17

URL: http://dx.doi.org/10.5430/crcp.v1n2p17

\begin{abstract}
Primary carcinoid tumor of the kidney is a very rare entity $(<1 \%)$. About 90 cases have been reported in the literature. Carcinoid tumors appear over-represented in horseshoe kidneys compared with normal renal anatomy. Herein, we reported a 33-year-old male with primary renal carcinoid tumor arising from a horseshoe kidney. The tumor showed nests and trabeculea of uniformly polygonal cells separated by vascular stroma. The cells have fine uniform nuclei and scanty to moderate amount of eosinophilic cytoplasm. No mitosis was seen. The tumor cells were positive for cytokeratin and neuroendocrine cell marker, synaptophysin, but negative for chromogranin A. To the best of our knowledge, this is the first case of primary renal carcinoid tumor arising from a horseshoe kidney in Egypt and the 19th in the literature.
\end{abstract}

\section{Key words}

Carcinoid tumor, Horseshoe kidney

\section{I ntroduction}

Carcinoid tumors are neuroendocrine neoplasms of low-grade malignant potential arising frequently within the gastrointestinal tract and respiratory tree. Primary carcinoid tumor of the kidney is a very rare entity $(<1 \%)^{[1]}$. About 90 cases have been reported in the literature ${ }^{[2]}$ since being first described by Resnick in $1966^{[3]}$.

Carcinoid tumors appear over-represented in horseshoe kidneys compared with normal renal anatomy ${ }^{[4]}$. Lanson et al. reported the first case of primary renal carcinoid tumor arising from a horseshoe kidney ${ }^{[5]}$. De Hoog et al. reviewed 17 cases of primary renal carcinoid tumors arising in a horseshoe kidney and described the 18th case ${ }^{[1]}$. Here, we described the 19th case of primary renal carcinoid occurring in a horseshoe kidney.

\section{Case presentation}

A 33-year-old male patient was incidentally discovered with left hypochondrial mass three months before hospital admission with irrelevant past and family history. General examination of the patient was free, however local examination 
revealed left renal mass. Serum creatinine was $0.8 \mathrm{mg} / \mathrm{dl}$. A contrast enhanced computed tomography (CT) scan of the abdomen demonstrated soft tissue renal mass related to the hilum of the left compartment of horseshoe kidney extending to the lower pole nearby its isthmus (see Figure 1). It measured about $(9.6 \mathrm{~cm} \times 9.2 \mathrm{~cm} \times 9.5 \mathrm{~cm}$ ) as regard width $\times$ length $\times$ height $(\mathrm{W} \times \mathrm{L} \times \mathrm{H})$. Peripheral hyper dense foci of calcification were identified in the lower part of the mass at pre-contrast phase. After IV contrast administration it exhibited mild progressive enhancement with multiple small areas of cystic degeneration. The mass was seen stretching left hilar renal vessels with enlarged left hilar and left para-aortic lymphadenopathy. Patient underwent left nephrectomy included left compartment and proximal part of renal isthmus via midline transperitoneal approach. Concomitant hilar lymphadenectomy was carried out. Macroscopically; the resected left compartment was with intact capsule apart from at the site of resection of horse shoe isthmus. The mass is well circumscribed, soft to firm in consistency and bright brown in color. The cut surface is cystic with wide area of hemorrhage and lymph node measured about $(0.5 \mathrm{~cm} \times 0.5 \mathrm{~cm})$ (see Figure 2). Microscopic examination revealed nests and trabeculae of uniformly polygonal cells separated by vascular stroma. The cells have fine uniform nuclei and scanty to moderate amount of eosinophilic cytoplasm. No mitosis was seen (see Figure 3A-C). The tumor cells were positive for cytokeratin and neuroendocrine cell marker, synaptophysin (see Figure 3D) but negative for chromogranin A.
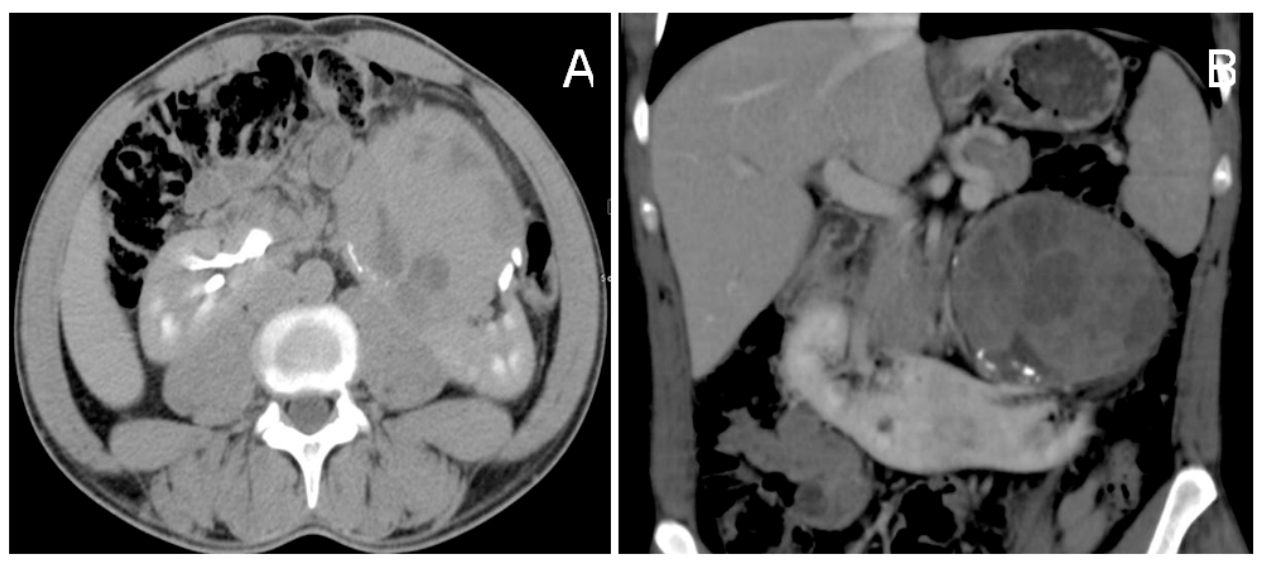

Figure 1. A. Axial contrast enhanced (CE) CT image. The corticomedullary phase demonstrates the close relation between the mass and left colon laterally, psoas muscle posteromedially, and pancreatic tail superomedially. No evidence of extracapsular extension. B. Coronal reconstructed CECT image shows compression of the hilum of left compartment of horse shoe kidney by the mass that extends inferiorly nearby the isthmus. Cystic areas are clearly identified inside the mass.

Figure 2. Gross picture of renal carcinoid. The mass was a well-circumscribed; light brown; soft to firm mass. The cut surface is cystic.

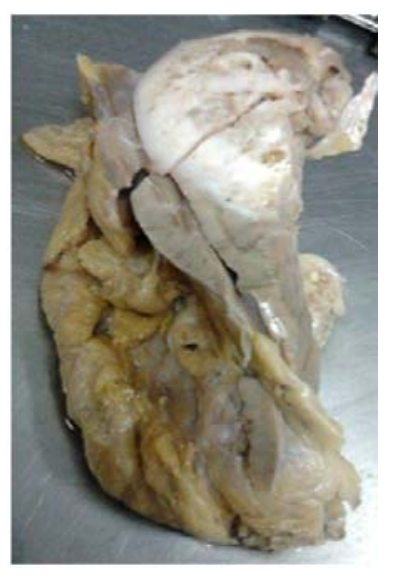

The post operative course was smooth. On retrospective evaluation, the patient didn't show any symptoms of carcinoid syndrome such as facial hot flushes and diarrhea. Postoperative colonoscopy and upper gastrointestinal endoscopy revealed no abnormality pertaining to gastrointestinal source of the tumor. No tumor recurrence was detected on close CT follows up. 
Figure 3. Histopathological features of carcinoid tumour. A. Carcinoid tumor is seen separated by capsule from renal cortex as evidenced by glomeruli (H\&E, $\times 40)$. B. Insular and trabecular growth pattern separated by vascular stroma $(H \& E, \times 100)$. C. trabecular growth pattern separated by hyalined stroma (H\&E, ×200). D. Positive cytoplasmic staining of tumour cells for synaptophysin (DAB $\times 400$ ).

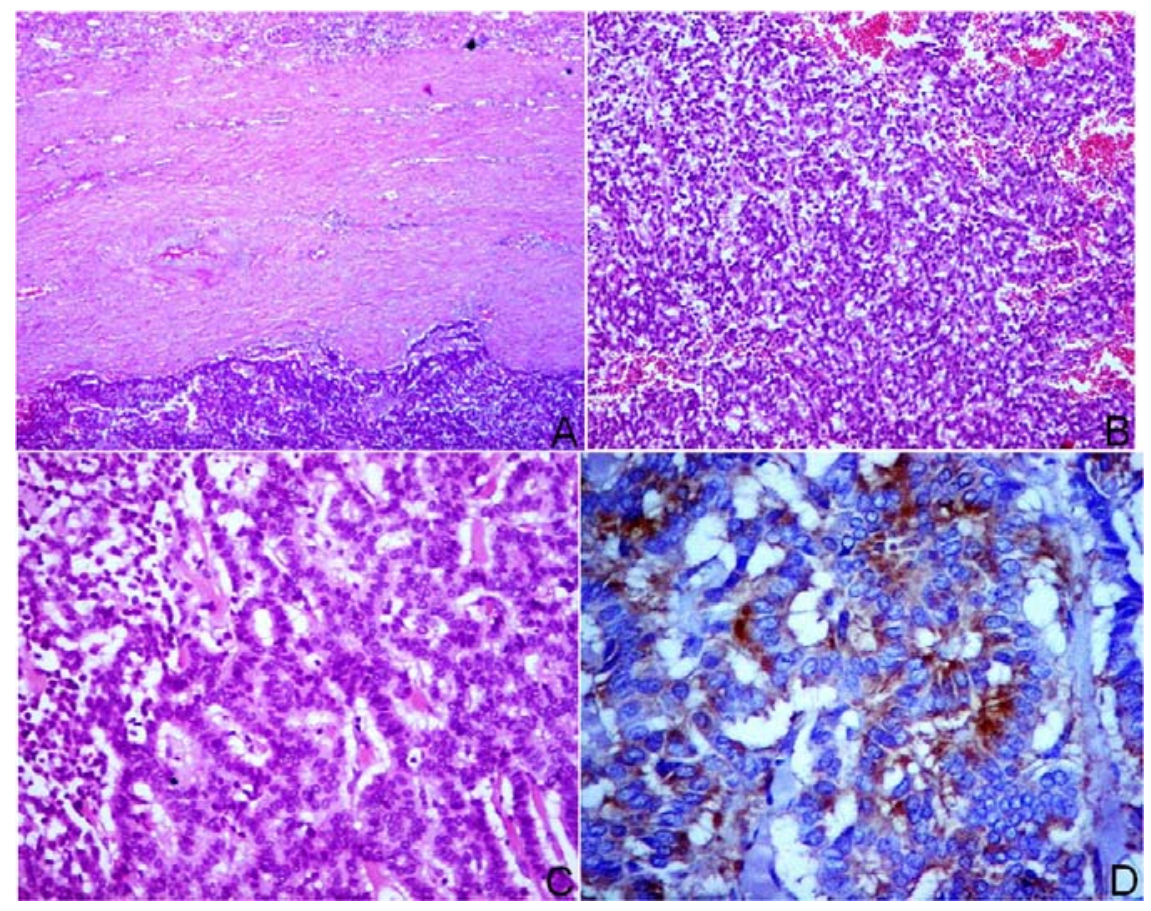

\section{Discussion}

Although rare, carcinoid tumour arising within the kidney is a well-defined entity. Lane et al. studied primary neuroendocrine tumours of the kidney and found only 56 published case reports of primary renal carcinoid, 10 of which arose within horseshoe kidneys ${ }^{[6]}$. Krishman et al. reported a relative risk of 62-fold for the occurrence of renal carcinoid in association with a horseshoe kidney ${ }^{[7]}$, while Bégin et al. estimated the relative risk for such an association to be 82fold ${ }^{[8]}$.

Neuroendocrine cells are not identified within the kidney or renal pelvis. So, the origin of renal carcinoids has been postulated to be derived from a primitive cell line that has the capacity to differentiate in a neuroendocrine direction ${ }^{[9]}$. Primary renal carcinoid tumors are more frequent with horseshoe kidneys, possibly because the existence of aberrant epithelium or teratomatous elements is more frequent in these kidneys ${ }^{[7]}$.

The clinical presentations of renal carcinoid tumor were nonspecific ${ }^{[10]}$. Renal carcinoid tumors can cause either localized symptoms, such as hematuria, abdominal pain, or flank pain, or systemic symptoms (carcinoid syndrome) such as perspiration and diarrhea. However, cases without any symptoms, as our case, are reported ${ }^{[11]}$.

Because carcinoid tumors do not show endocrinologic symptoms, it is difficult to diagnose and predict the existence of these tumors by the results of blood tests ${ }^{[11]}$. Also, the image features of renal carcinoid tumors resemble in appearance typical tumors inside the kidney such as renal cell carcinoma ${ }^{[11]}$. So, Carcinoid tumors are generally diagnosed through pathological examination after surgery. That is why we did not do blood tests as 5-hydroxy indole acetic acid (5-HIAA) or OctreoScan.

Carcinoid tumors are usually well circumscribed, tan to yellow, solid fleshy tumors with areas of necrosis and cystic degeneration ${ }^{[12]}$. Microscopically, Primary carcinoid tumors of the kidney are similar to those in other sites of the body. Renal carcinoids are characterised by tumor cells are arranged in ribbons, festoons, and solid nests ${ }^{[12]}$. The neoplastic cells have eosinophilic granular cytoplasm and nuclei containing fine stippled chromatin ${ }^{[1]}$. Mitotic activity and vascular invasion are rare ${ }^{[12]}$. 
Nearly all lesions reported are positive for synaptophysin and chromogranin1 which is slightly different from our case as it was negative for chromogranin and positive for synaptophsin. The diagnosis of carcinoid tumor is initially based on histology with confirmation by positive immunohistochemical staining, defined as positive staining for one or more of neuroendocrine markers (such as chromogranin A or synaptophysin) ${ }^{[13]}$.

Our case represented with lymph node metastasis. Isobe presented a case with accompanying lymph node metastasis, which had no evidence of local recurrence or distant metastasis for three years after surgery ${ }^{[14]}$. Radical nephrectomy with adjacent lymph node dissection is the treatment of choice because renal carcinoid tumors have a reported distant metastasis. If the mass is small and confined to the kidney, partial nephrectomy may be possible ${ }^{[11]}$.

Usually, carcinoid lesions in the kidneys exhibit a less aggressive biologic behavior than renal cell carcinoma ${ }^{[12]}$. However, the biologic behavior of primary renal carcinoid tumor arising within a horseshoe kidney appears to be less aggressive than that of the non-horseshoe variant ${ }^{[7]}$. All these patients were alive three years after complete or partial nephrectomy with no evidence of disease ${ }^{[15]}$.

In summary, we have described a case of a 33-year-old man with primary renal carcinoid tumor arising from a horseshoe kidney. To the best of our knowledge, this is the first case in Egypt and the 19th in the literature.

\section{References}

[1] De Hoog JP, Murray S, Chou W. Horseshoe kidney and primary renal carcinoid tumour: a case report of a rare entity. Grand Rounds. 2010; 10: 46-50.

[2] Jain D, Sharma MC, Singh K, Gupta NP. Primary renal carcinoid tumors: case report and brief review of literature. Indian J Pathol Microbiol. 2010; 53: 772-4. PMid: 21045413. http://dx.doi.org/10.4103/0377-4929.72087

[3] Resnick ME, Unterberger H, McLoughlin PT. Renal carcinoid producing the carcinoid syndrome. Med Times. 1966; 94: 895-6. PMid: 5938842.

[4] Shurtleff BT, Shvarts O, Rajfer J. Carcinoid tumor of the kidney: case report and review of the literature. Rev Urol. 2005; 7: 229-33. PMid: 16985835.

[5] Lanson Y, Bruant D, Benatre A, Rivalan P, Brizon J. Carcinoid tumor of the kidney. One case (author's transl). J Urol Nephrol. 1978; 84: 47-51.

[6] Lane BR, Chery F, Jour G, Sercia L, Magi-Galluzzi C, Novick AC, et al. Renal neroendocrine tumours: a clinicopathological study. BJU Int. 2007; 100: 1030-5. PMid: 17784891.

[7] Krishnan B, Truong LD, Saleh G, Sirbasku DM, Slawin KM. Horseshoe kidney is associated with an in creased relative risk of primary renal carcinoid tumor. J Urol. 1997; 157: 2059-66. http://dx.doi.org/10.1016/S0022-5347(01)64674-3

[8] Bégin LR, Guy L, Jacobson SA, Aprikian AG. Renal carcinoid and horseshoe kidney: a frequent association of two rare entities a case report and review of the literature. J Surg Oncol. 1998; 68:113-9. http://dx.doi.org/10.1002/(SICI)1096-9098(199806)68:2<113::AID-JSO8>3.0.CO;2-9

[9] Cauley JE, Almagro UA, Jacobs SC. Primary renal carcinoid tumor. Urology. 1988; 32: 564-6. http://dx.doi.org/10.1016/S0090-4295(98)90046-2

[10] Chen CH, Pu YS, Chen J, Huang CY. Renal Carcinoid Tumor with Hemorrhage in a Patient with Breast Cancer: A Case Report. JTUA. 2004; 15 (4): 180-183.

[11] Seo HP, Shin DE, Lim DH, Muhn HY, Kim CS. Primary Renal Carcinoid Tumor. Korean Journal of Urology. 2009; (50) $293-295$.

[12] Rafique M. A Primary Carcinoid Tumor of Kidney. Urol J. 2008; 5: 60-1. PMid: 18454431.

[13] Pincho SNt, Holen K, Sippel KS, Chen H. Carcinoid Tumors. The Oncologist. 2008; 13 (12): 1255-1269. PMid: 19091780. http://dx.doi.org/10.1634/theoncologist.2008-0207

[14] Isobe H, Takashima H, Higa shi N, Murakami Y, Fujita K, Hanazawa K, et al. Primary carcinoid tumor in a horseshoe kidney. Int J Urol. 2000; 7:184-8. PMid: 10830826. http://dx.doi.org/10.1046/j.1442-2042.2000.00160.x

[15] Tal R, Lask DM, Livne AM. Metastatic renal carcinoid. Case report and review of literature. Urology. 2003; 61: 838xv-838xvii. 